\title{
The time-varying geomagnetic field of Southern Africa
}

\author{
P. B. Kotzé \\ Hermanus Magnetic Observatory, South Africa \\ (Received October 25, 2002; Revised February 20, 2003; Accepted March 8, 2003)
}

\begin{abstract}
The geomagnetic field at any given epoch is a function of space coordinates, varying differently at each location with time. It has been known that secular change is a comparatively local phenomenon and that it does not proceed in a regular way all over the Earth, giving rise to regions where the field changes more rapidly than elsewhere, like for instance southern Africa. The Hermanus Magnetic Observatory routinely executes geomagnetic repeat surveys, which includes South Africa, Namibia, Zimbabwe and Botswana. Spherical cap modelling of field survey and observatory secular variation data at 5 year intervals between 1975 and 2000 shows that a geomagnetic jerk occurred between 1980 and 1985 over southern Africa. The secular variation models are based on 70 repeat station data central differences as well as the 3 magnetic observatories at Hermanus, Hartebeesthoek and Tsumeb (Namibia) and include terms up to spatial degree 3 and temporal degree 2. Although each model allows for 48 coefficients, only 42 were found to be statistically significant.
\end{abstract}

\section{Introduction}

The geomagnetic field of the Earth varies on timescales ranging from seconds to millions of years. Variations on short timescales are mostly dominated by external sources, while variations on longer timescales ( $\sim 1$ year and longer) are collectively known as secular variation and are predominantly of internal origin. It is also known that secular change is a comparatively local phenomenon and does not proceed in a regular way all over the Earth (Bullard, 1948), giving rise to regions where the field changes more rapidly than elsewhere, as is the case for southern Africa. The observed high secular variation in southern Africa has been ascribed by Bloxham and Gubbins (1985) to rapidly drifting core spots. These intense patches normally occur in the Southern Hemisphere and drift westwards towards South America.

Several localised regions where abrupt changes in the secular variation have occurred are known to date. Mizuno (1980) examined the variation in the annual mean value of the north component of the Earth's magnetic field from 1964 to 1976 at the Kakioka Magnetic Observatory and observed abrupt changes in the secular variation rate around 1965 and 1974. Neither of these impulses was of global distribution, showing that regional abrupt changes in the secular variation rate with a time scale of 1 year do exist.

During 1983/1984 an abrupt secular variation change occurred in the southern African subcontinent where the Hermanus Magnetic Observatory routinely executes geomagnetic repeat surveys, including countries such as South Africa, Namibia, Botswana and Zimbabwe. A map showing the location of the various field stations can be seen in Fig. 1.

The phenomenon was first observed in the data from the

Copy right(C) The Society of Geomagnetism and Earth, Planetary and Space Sciences (SGEPSS); The Seismological Society of Japan; The Volcanological Society of Japan; The Geodetic Society of Japan; The Japanese Society for Planetary Sciences. continuous recording stations at Hermanus, Hartebeesthoek and Tsumeb (Namibia), and was subsequently confirmed in results from regional surveys at epochs 1980, 1985, 1990, 1995 and 2000 as shown in Fig. 2. During the period 1970 till 1984 the D secular variation at Hermanus was determined at a constant rate of $5 \mathrm{~min}$./year, whereas during 1983/85 it changed abruptly to $-2.7 \mathrm{~min}$./year, constituting a change from predominantly eastward to westward. A similar trend was observed at Hartebeesthoek, where the secular variation in D changed from $6.3 \mathrm{~min}$./year during 1970-1985, to $-3.3 \mathrm{~min}$./year after 1985 . At Tsumeb, however only a slight change in the declination secular variation could be observed, where it changed from $11.7 \mathrm{~min}$./year to 9.3 min./year during the period under discussion. It is also apparent from Fig. 2 that another change in secular variation took place at Hermanus and Hartebeesthoek and Tsumeb during 1993/1994. At Hermanus the secular variation in D changed from $-2.7 \mathrm{~min}$./year to a value of $-4.1 \mathrm{~min} . / \mathrm{year}$, while at Hartebeesthoek the change was from $-3.3 \mathrm{~min} . /$ year to $-4.6 \mathrm{~min}$./year. At Tsumeb, however again only a slight change in the secular variation of the $\mathrm{D}$ component could be detected from $9.3 \mathrm{~min}$./year to $7.8 \mathrm{~min}$./year, maintaining its eastward direction.

\section{Data Collection and Field Surveys}

Continuous recording of geomagnetic field variations are conducted at Hermanus $\left(34^{\circ} 25.5^{\prime} \mathrm{S}, 19^{\circ} 13.5^{\prime} \mathrm{E}\right)$, Hartebeesthoek $\left(25^{\circ} 52.9^{\prime} \mathrm{S}, 27^{\circ} 42.4^{\prime} \mathrm{E}\right)$ and Tsumeb $\left(19^{\circ} 12^{\prime} \mathrm{S}\right.$, $\left.17^{\circ} 35^{\prime} \mathrm{E}\right)$. The primary instrument for recording of magnetic field variations is the FGE fluxgate magnetometer, manufactured by the Danish Meteorological Institute in Copenhagen, Denmark. This instrument is based on three-axis linear-core fluxgate technology, optimised for long-term stability and records the components $\mathrm{H}, \mathrm{D}$ and $\mathrm{Z}$. Another system also in operation at all three stations has an integrated design for 


\section{Southern Africa : Field Survey (2000.0)}

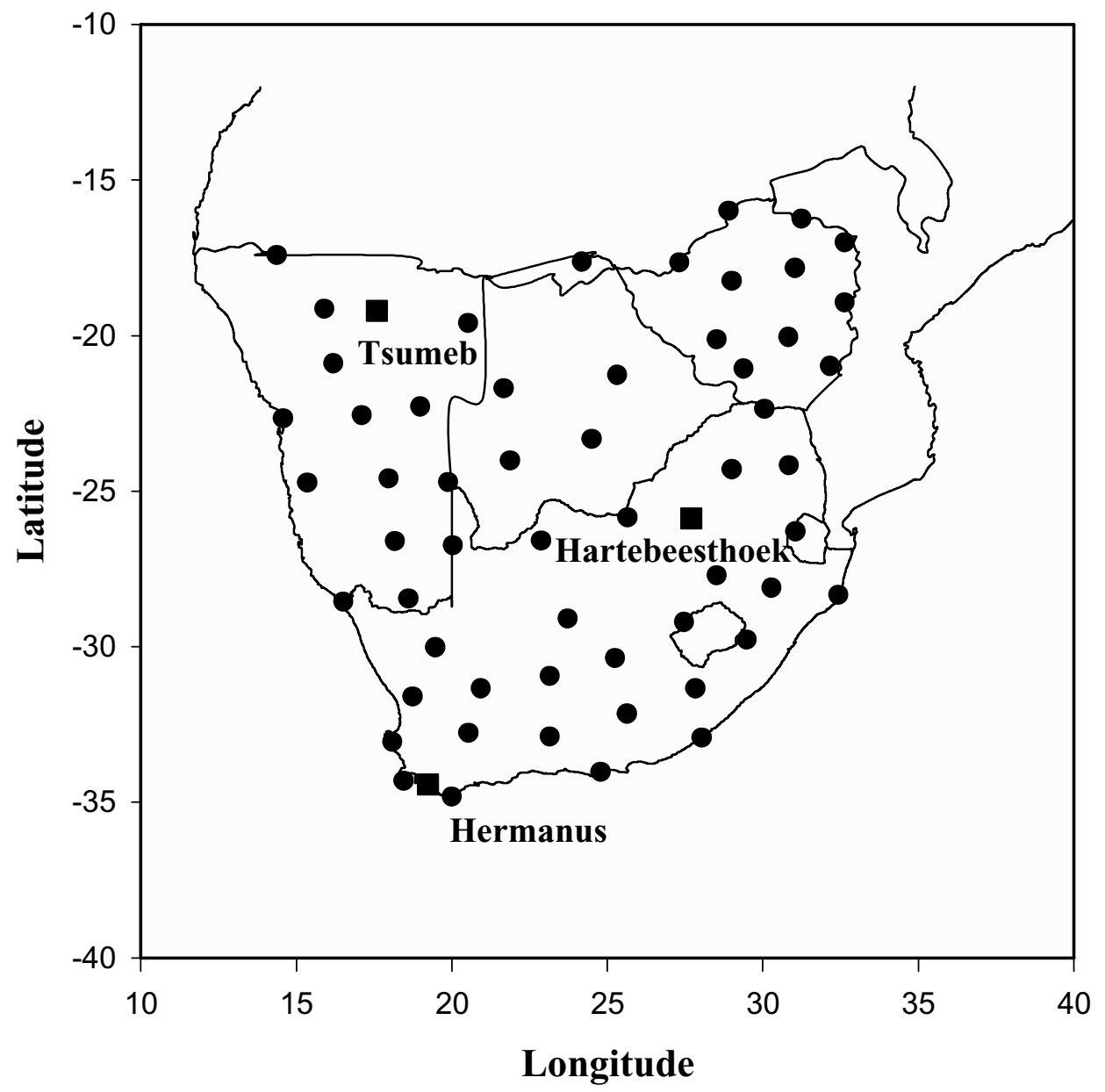

Fig. 1. A map showing the various field survey stations (indicated by Hermanus, Hartebeesthoek and Tsumeb (indicated by $\mathbf{\square}$ ).

measuring the Earth's magnetic field by a sequence of firstly measuring the total magnetic field and then four biased values of the magnetic field with a Geometrics sensor mounted at the centre of a set of coils in order to obtain F, H, D and Z.

For field survey purposes, field stations are marked by concrete beacons, ensuring that all observation points are exactly reoccupied during surveys. All measurements are taken on a standard $1.2 \mathrm{~m}$ pillar. A DI fluxgate magnetometer was used as primary instrument during field surveys to obtain values of D and I, while a Geometrics PPM instrument delivered values of total field intensity (F). During field surveys a Quartz Horizontal Force magnetometer was employed to obtain the horizontal intensity $(\mathrm{H})$. In order to reduce the influence of the geomagnetic diurnal variation, observations were carried out during the period 1800-2000 UT, necessitating the use of a special non-magnetic fluorescent lamp in the observation tent.

In our investigation we used only repeat stations that were occupied during all surveys conducted during 1975, 1980, 1985, 1990, 1995 and 2000. Corrections for diurnal variation and other disturbing effects were made by comparing field station observations with magnetic data obtained at the continuous recording observatories, Hermanus, Hartebeesthoek and Tsumeb. This was done by comparing repeat station observations with the closest magnetic observatory during field surveys, thereby ensuring that applicable diurnal variations are taken into account. Annual mean values from these observatories were obtained using only quiet daily mean values. The field survey measurements were subsequently reduced to a common epoch, by interpolating between the preceding survey and the present survey. For a particular magnetic field component the differential secular variation at a field station relative to a control observatory is calculated by assuming a linear drift between different survey epochs.

Since the surveys were conducted during 1975, 1980, 1985, 1990, 1995 and 2000, the time span for reduction of data amounts to less than 1 year.

\section{Modelling of Field Survey Data}

Spherical Cap Harmonic Analysis (Haines, 1985a) was used to model the field survey data sets between 1975 and 2000. Spherical Cap Harmonic Analysis (SCHA) is a mathematical technique developed specifically to model a potential field and its spatial derivatives, or a general function and its surface derivatives, on a regional scale in order to overcome the non-orthogonality problem in the case of global spheri- 


\section{Hermanus, Hartebeesthoek \& Tsumeb Annual Mean Declination}

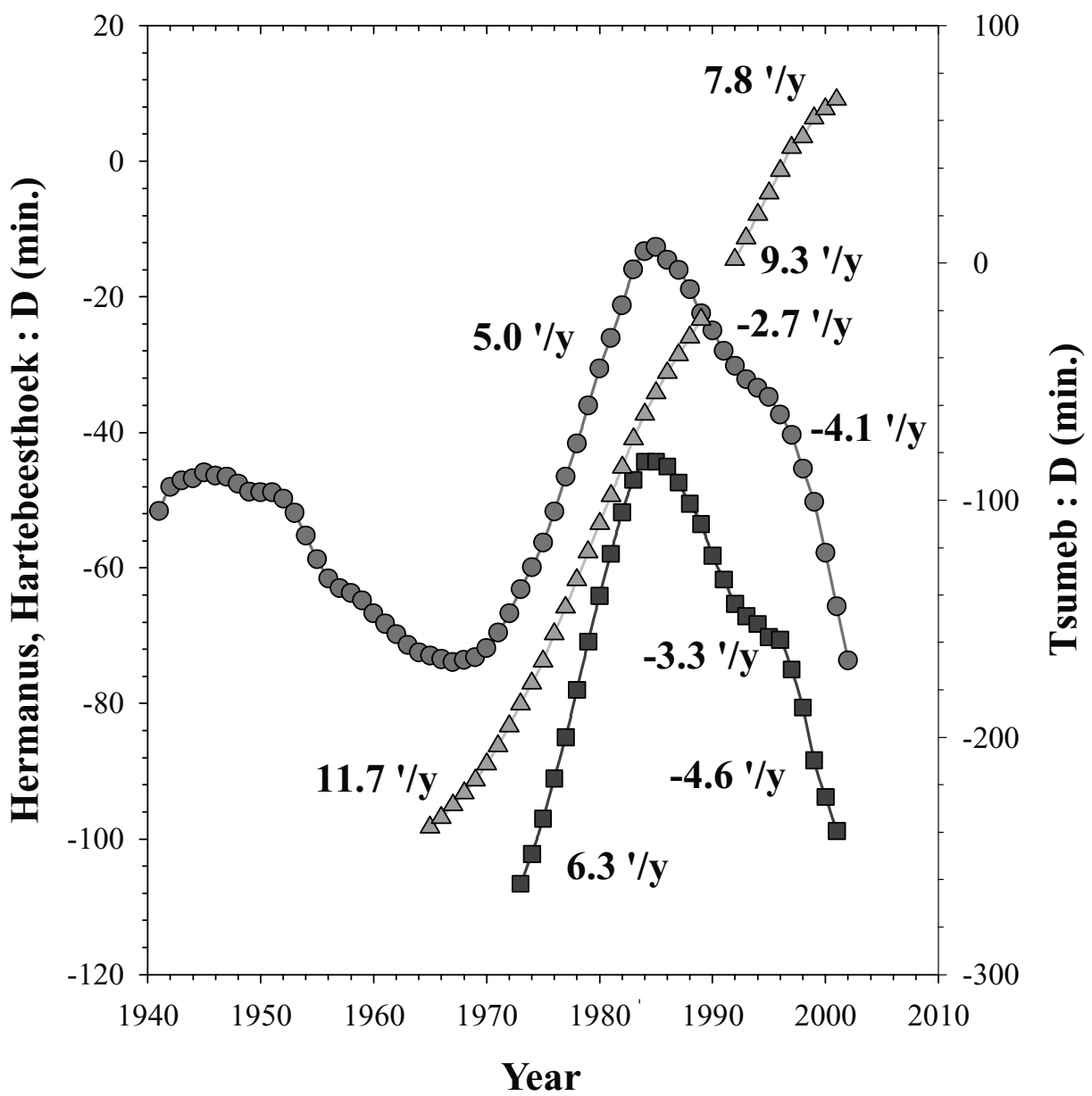

$$
\begin{aligned}
& - \text { Hermanus : -23 Deg. }+ \text { D } \\
& \square-\text { Hartebeesthoek : }-15 \text { Deg. }+ \text { D } \\
& \triangle \quad \text { Tsumeb : }-12 \text { Deg. }+ \text { D }
\end{aligned}
$$

Fig. 2. A plot showing the secular variation in declination (D) as observed at Hermanus, Hartebeesthoek and Tsumeb.

cal harmonic models when applied to restricted areas. The SCHA modelling technique has also been used successfully to derive a regional field model over southern Africa, using vector data from the Ørsted satellite mission (Kotzé, 2001). By making every harmonic coefficient a polynomial in time, a SCHA model provides both spatial and temporal continuity, enabling the modelling of secular variation as well. This was successfully employed by Haines (1985b) to derive a SCHA model of geomagnetic secular variation over Canada.

The solution to Laplace's equation over a spherical cap for both internal as well as external sources is given by:

$$
V(r, \theta, \lambda)=a \sum_{k=0}^{K \text { int }} \sum_{m=0}^{k}\left(\frac{a}{r}\right)^{n_{k}(m)+1} P_{n_{k}(m)}^{m}(\cos \theta)
$$

$$
\begin{aligned}
& *\left[g_{k}^{m, i}(t) \cos (m \lambda)+h_{k}^{m, i}(t) \sin (m \lambda)\right] \\
& +a \sum_{k=1}^{K \operatorname{ext}} \sum_{m=0}^{k}\left(\frac{r}{a}\right)^{n_{k}(m)} P_{n_{k}(m)}^{m}(\cos \theta) \\
& *\left[q_{k}^{m, e} \cos (m \lambda)+h_{k}^{m, e} \sin (m \lambda)\right]
\end{aligned}
$$

where:

$t$ is a time or epoch;

$r, \theta, \lambda$ are the geocentric spherical coordinates radius, colatitude and longitude;

$a=$ reference radius;

$P_{n_{k}(m)}^{m}(\cos \theta)=$ associated Legendre function with integral order $m$ and real degree $n_{k}(m)$;

$k=$ ordering index, with $K$ int the maximum index for internal sources, and $K$ ext the maximum index for exter- 
nal sources;

$$
\begin{aligned}
& g_{k}^{m, i}(t)=\sum_{q=0}^{Q} g_{k, q}^{m} t^{q} \\
& h_{k}^{m, i}(t)=\sum_{q=0}^{Q} h_{k, q}^{m} t^{q}
\end{aligned}
$$

are the internal spherical cap coefficients, with $Q$ the parameter that determines the amount of temporal variation permitted; with $q_{k}^{m, e}, h_{k}^{m, e}$ the external spherical cap coefficients.

If the half-angle of the spherical cap is denoted by $\theta_{0}$, the $n_{k}(m)$ are determined as the roots of the equation, for given $m$ :

$$
d P_{n_{k}(m)}^{m}\left(\cos \theta_{0}\right) / d \theta=0, \quad k-m=\text { even }
$$

and additionally, if differentiability with respect to $\theta$ is required:

$$
P_{n_{k}(m)}^{m}\left(\cos \theta_{0}\right)=0, \quad k-m=\text { odd } .
$$

If the expansion in Eq. (1) is truncated at $k=K$, and $q=Q$, the number of model coefficients is $(K+1)^{2}(Q+1)$. These coefficients are determined by least-squares and define any particular model.

Since secular variation is not measured directly, but is derived as a time derivative of the geomagnetic field, one can either model the main field and then differentiate the corresponding field model to get a secular variation model, or one can numerically differentiate the main field data and then fit a secular variation model directly. The latter derivative-fit approach is able to remove crustal contamination and has been applied in this study of observatory and repeat station data. First central differences from annual mean observatory data as well as repeat stations, divided by their respective time intervals in years, were used as input data to the secular variation model of Eq. (1). The data were subsequently converted from geodetic to geocentric coordinates, after which this geocentric coordinate system was transformed to a new pole at $27^{\circ} \mathrm{S}, 25^{\circ} \mathrm{E}$.

A half-cap angle of $20^{\circ}$ provided a best fit to data for all surveys. The maximum index $K$ was taken as 3 , and the maximum degree $Q$ of the polynomial in time was taken as 2. Although there are 48 coefficients in this spherical cap harmonic expansion, only 42 coefficients tested statistically significant at an F-level of 4.

Although observatory data in general are more accurate than repeat survey data, because of better baseline control and because seasonal and other short-term variations are removed by using annual means, we did not introduce any subjective weighing factors. Both observatory and repeat station secular variation data were weighted equally in the leastsquares solution. There were 210 vector differences from 70 repeat stations and 9 vector differences from the $3 \mathrm{ob}-$ servatories, providing a total of 219 data values for each 5year interval from 1975 till 2000. A secular variation model for each 5-year period was subsequently derived. The leastsquares routine used to fit the data was the stepwise regression procedure described by Efroymson (1960), which has the ability of both entering and removing variables at given levels of statistical significance. External fields were modelled separately at each interval, limiting the expansion to $K=2$, and were subsequently subtracted from the data prior to deriving the secular variation models. The scatter about the fit for declination secular variation was less than $1 \mathrm{~min}^{-1} / \mathrm{y}$.

The maximum degree used in all secular variation spherical cap harmonic models is 15.3 , and with an average distance of $350 \mathrm{~km}$ between repeat stations, spatial aliasing can be considered as negligible.

\section{Discussion and Conclusions}

Studies of geomagnetic data obtained from observatories worldwide have revealed numerous sudden changes in the trend of the secular variation which have been named 'geomegnetic jerks' or 'secular variation impulses' (Alexandrescu et al., 1995, 1996). The internal origin of these jerks seems to be accepted (Malin and Hodder, 1982). Geomagnetic jerks have been investigated and discussed by various authors (Courtillot and Le Mouël, 1984; McLeod, 1985, 1989; Golovkov et al., 1989; Stewart and Whaler, 1992; Le Huy et al., 1998, 2000). These analyses have revealed the predominantly worldwide character and internal origin of these events.

It is evident from Fig. 2 that the 1969/1970 worldwide event clearly shows in the Hermanus data. The event observed in southern Africa around 1984/1985 however does not seem to be reported elsewhere. Although the characteristics of the geomagnetic impulse at Hermanus and Hartebeesthoek show strong similarities, it is not the case at Tsumeb, where the secular variation in declination continues to be in an eastward direction. Similar tendencies could also be detected at other surrounding repeat stations in Namibia, e.g. Windhoek where the secular variation changed from $10.6 \mathrm{~min}$./year before 1985 to $6.3 \mathrm{~min}$./year during 1985 1990.

The results obtained at the 3 continuous recording magnetic observatories, Hermanus, Hartebeesthoek and Tsumeb, are confirmed by modelling geomagnetic secular variation data over southern Africa by spherical cap harmonic analysis, as displayed in Fig. 3. Results obtained prior to 1980 show that the secular variation for declination over southern Africa is predominantly in an eastward direction, except for a small eastern coastal region. During 1980-1985 this pattern changed abruptly, showing an eastward tendency in the northern parts of the subcontinent, while the southern region obtained a westward secular variation characteristic. This tendency continued during 1990-1995 as well as 19952000

Another global sudden change in the secular variation of the geomagnetic field has been reported around 1991 (MacMillan, 1996; De Michelis et al., 1998). This tendency has also been observed at Hermanus, Hartebeesthoek and Tsumeb during the early 1990's. It is also evident from Fig. 2 that since 1995 an acceleration process has been taking place in the secular variation for declination as observed at both Hermanus and Hartebeesthoek. In 1996 the observed secular variation at Hermanus and Hartebeesthoek were -2.6 $\mathrm{min} . /$ year and $-1.9 \mathrm{~min}$./year respectively, while at present 


\section{Declination Secular Variation $(\mathrm{min} / \mathrm{y})$}
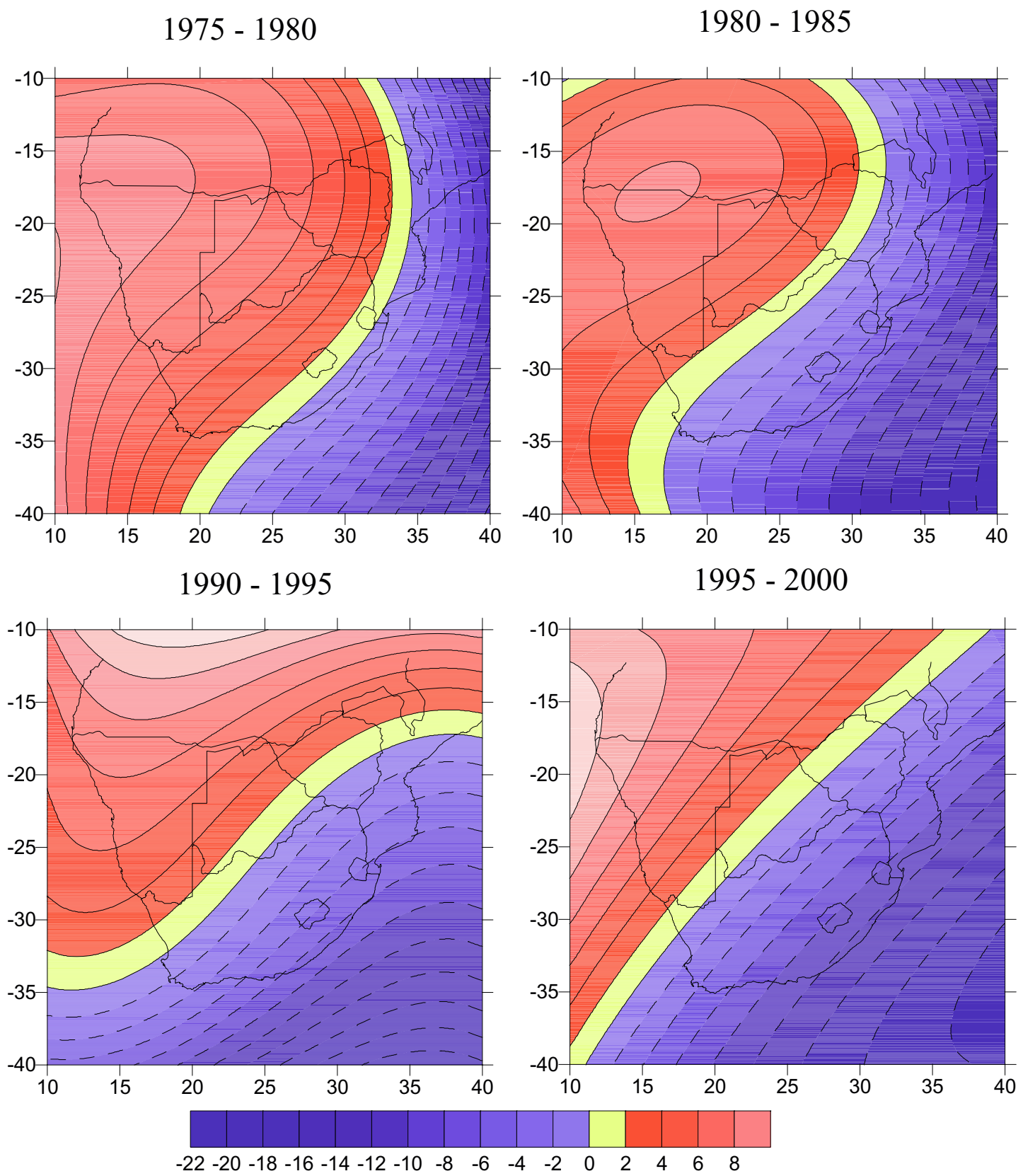

Fig. 3. A plot showing the declination secular variation over southern Africa as modelled by Spherical Cap Harmonic Analysis for various time intervals between 1975 and 2000. Variations are between $-20 \mathrm{~min} / \mathrm{y}$ and $14 \mathrm{~min} / \mathrm{y}$. Negative secular variation (westward) is indicated by blue, while positive secular variation (eastward) is indicated by red. The region of zero secular variation is indicated by yellow.

it is $-7.9 \mathrm{~min}$./year at Hermanus and $-8.6 \mathrm{~min}$./year at Hartebeesthoek. In contrast, however the secular variation as observed at Tsumeb shows no such behaviour. The consequence of this pattern is that the gradient in declination over southern Africa is increasing with time. It is also evident that if this tendency continues, we will quite soon be able to measure the largest declination value at Hermanus since its founding in 1941 .

Acknowledgments. We thank Louis Loubser for the provision of high-quality data as well as Hisayoshi Shimizu and Michael Pu- rucker for their helpful comments on an earlier version of the manuscript.

\section{References}

Alexandrescu, M., D. Gibert, D. Hulot, J.-L. Le Mouël, and G. Saracco, Detection of geomagnetic jerks using wavelet analysis, J. Geophys. Res., 100, 12557-12572, 1995.

Alexandrescu, M., D. Gibert, D. Hulot, J.-L. Le Mouël, and G. Saracco, Worldwide wavelet analysis of geomagnetic jerks, J. Geophys. Res., 101, 21975-21994, 1996.

Bloxham, J. and D. Gubbins, The secular variation of Earth's magnetic field, Nature, 317, 777-781, 1985. 
Bullard, E. C., The secular change in the Earth's magnetic field, Mon. Notices Royal Astronom. Soc., Geophys. Suppl., 5, 248-257, 1948.

Courtillot, V. and J.-L. Le Mouël, Geomagnetic secular variation impulses: A review of observational evidence and geophysical consequences, $\mathrm{Na}$ ture, 311, 709-716, 1984.

De Michelis, P., L. Cafarella, and A. Meloni, Worldwide character of the 1991 jerk, Geophys. Res. Lett., 25, 377-380, 1998.

Efroymson, M. A., Multiple regression analysis, in Mathematical Methods for Digital Computers, edited by A. Ralston and H. S. Wilf, pp. 191-203, John Wiley, New York, 1960.

Golovkov, V. P., T. I. Zvereva, and A. O. Simonyan, Common features and differences between 'jerks' of 1947, 1958 and 1969, Geophys. Astrophys. Fluid Dyn., 49, 81-96, 1989.

Haines, G. V., Spherical cap harmonic analysis, J. Geophys. Res., 90, $2583-$ 2591, 1985a.

Haines, G. V., Spherical cap harmonic analysis of geomagnetic secular variation over Canada 1960-1983, J. Geophys. Res., 90, 12563-12574, 1985b.

Kotzé, P. B., Spherical cap modelling of Ørsted magnetic field vectors over southern Africa, Earth Planets Space, 53, 357-361, 2001.

Le Huy, M., M. Mandea, G. Hulot, and J.-L. Le Mouël, On the characteris- tics of successive geomagnetic jerks, Earth Planets Space, 50, 723-732, 1998.

Le Huy, M., M. Mandea, J.-L. Le Mouël, and A. Pais, Time evolution of the fluid flow at the top of the core, Earth Planets Space, 52, 163-173, 2000 MacMillan, S., A geomagnetic jerk for the early 1990's, Earth Planet. Sci. Lett., 137, 189-192, 1996.

McLeod, M. G., On the geomagnetic jerk of 1969, J. Geophys. Res., 90, 4597-4610, 1985.

McLeod, M. G., Geomagnetic secular variation, in Geomagnetism and Paleomagnetism, edited by F. J. Lowes, D. W. Collison, J. H. Parry, S. K Runcorn, D. C. Tozer, and A. Soward, pp. 19-30, Kluwer Acad., Mass., 1989.

Malin, S. R. C. and B. M. Hodder, Was the 1970 geomagnetic jerk of internal or external origin?, Nature, 296, 726-728, 1982.

Mizuno, H., Abrupt change in secular variation rate of geomagnetic north component observed in Japan, J. Geomag. Geoelectr., 32, 413-420, 1980. Stewart, D. N. and K. Whaler, Geomagnetic disturbance fields: An analysis of observatory monthly means, Geophys. J. Int., 108, 215-223, 1992.

P. B. Kotzé (e-mail: pkotze@hmo.ac.za) 